\title{
Magnitude of Dopamine Release in Medial Prefrontal Cortex Predicts Accuracy of Memory on a Delayed Response Task
}

\author{
Anthony G. Phillips, ${ }^{1 \star}$ Soyon Ahn, ${ }^{1 \star}$ and Stan B. Floresco ${ }^{2 \star}$ \\ ${ }^{1}$ Department of Psychiatry and Brain Research Center, University of British Columbia, Vancouver, British Columbia, Canada, V6T 2A1, and ${ }^{2}$ Department of \\ Psychology and Brain Research Center, University of British Columbia, Vancouver, British Columbia, Canada, V6T 1Z4
}

\begin{abstract}
Modulation of neural function in the prefrontal cortex (PFC) by dopamine (DA) is essential for higher cognitive processes related to attention, working memory, and planning of future behavior. The present study demonstrates that DA efflux in the PFC is increased in a phasic manner when a rat engages in search behavior for food reward on an eight arm radial maze guided by memory, independent of whether or not reward is obtained for making the correct choice. Furthermore, disruption of accurate recall of the correct pattern of arms induced by increasing the delay period from $30 \mathrm{~min}$ to $1 \mathrm{or} 6 \mathrm{hr}$, is associated with attenuated DA efflux during the retrieval phase of the task. The observed increase in DA efflux in the absence of reward at a 30 min delay and the minimal increase during consumption of the same quantity of food during poor performance after an unexpected $6 \mathrm{hr}$ delay, argue against a simple relationship between DA function in the PFC and reward processes. Instead, these data demonstrate a close functional relationship between the release of DA from terminals within the PFC and the retrieval of specific trial unique memories; furthermore, the magnitude of mesocortical DA efflux is predictive of the accuracy of this form of memory.
\end{abstract}

Key words: dopamine; microdialysis; radial arm-maze; spatial memory; rat; reward

\section{Introduction}

Working memory functions subserved by the frontal lobes are highly dependent on mesocortical dopamine (DA) transmission (Williams and Goldman-Rakic, 1995; Robbins, 2000). Electrophysiological recordings in freely behaving rats and primates have shown that neural activity in the prefrontal cortex (PFC) is positively correlated with the accuracy of behavior guided by working memory (Goldman-Rakic, 1992; Goldman-Rakic, 1995; Pratt and Mizumori, 2001) and that this activity is regulated by $\mathrm{DA} \mathrm{D}_{1}$ receptors (Williams and Goldman-Rakic, 1995). Pharmacological blockade of $\mathrm{DA} \mathrm{D}_{1}$ receptors in the PFC disrupts choice behavior guided by working memory, after both short (Sawaguchi et al., 1990; Sawaguchi and Goldman-Rakic, 1994) and long delays (Seamans et al., 1998). Furthermore, it has been proposed that working memory function is optimized when $\mathrm{DA} \mathrm{D}_{1}$ receptor occupancy is within a critical range of an inverted U-shaped function (Zahrt et al., 1997; Arnsten, 1998; Floresco and Phillips, 2001). Increased DA efflux in the primate PFC has been observed during repeated correct performance of a brief $10 \mathrm{sec}$ delayed alternation task (Watanabe et al., 1997). These findings indicate a general role for DA in working memory; however, it remains to

\footnotetext{
Received Aug. 1, 2003; revised Nov. 18, 2003; accepted Nov. 20, 2003.

This work was supported by a grant from the Natural Sciences and Engineering Research Council of Canada (A.G.P.).S.B.F. is a recipient of a Human Frontiers Science Organization postdoctoral fellowship. We thank Dr. Jeremy Seamans for helpful suggestions, Dr. A. Coury for her assistance with microdialysis, and E. Falconer, K. Brar, A. Valji, and Christina Cheng for their assistance with behavioral testing

${ }^{*}$ A.G.P., S.A., and S.B.F. all contributed equally to this work.

Correspondence should be addressed to Anthony G. Phillips at the above address. E-mail: aphillips@psych.ubc.ca.

D0I:10.1523/JNEUROSCI.4653-03.2004

Copyright $\odot 2004$ Society for Neuroscience $\quad 0270-6474 / 04 / 240547-07 \$ 15.00 / 0$
}

be determined whether phasic changes in mesocortical DA efflux in the medial PFC (mPFC) occur during different phases (i.e., acquisition, delay, and retrieval) of a delayed response task. Further evidence for dopaminergic modulation of memory processes mediated by the frontal lobes could be provided by in vivo measures of DA efflux in the PFC during different phases of delayed memory task.

The present study used in vivo microdialysis to monitor changes in DA release in the mPFC of rats performing a delayed response task on a radial-arm maze, using a delay period comparatively longer than previous studies $(30 \mathrm{~min})$. Unlike many other delayed-response tasks, this protocol does not depend on the component of working memory related to short-term (10-60 sec) retention of information, mediated by local PFC circuits (Goldman-Rakic, 1995). In this regard it is important to note that a broader definition of working memory, originally put forth by Baddeley (1986), refers to a collection of processes that include the temporary storage of information, as well as executive functions that mediate the manipulation and retrieval of trial-unique information to guide action after both short (seconds) and longer (minutes to hours) delays (Mizumori et al., 1987; Baddeley, 1992; Floresco and Phillips, 2001). In the present task (Fig. 1) information concerning the future location of food reward is obtained by a rat during a discrete acquisition phase, and memory of this trial-unique information is used to locate food accurately in a retention phase (Seamans et al., 1998). Behavioral studies using reversible disconnection lesions have confirmed that this type of delayed memory task is subserved by a neural circuit linking the ventral hippocampus to the mPFC (Floresco et al., 1997, 1999). Blockade of $\mathrm{DA}_{1}$ receptors in the $\mathrm{mPFC}$ during retrieval, but not acquisition, selectively disrupts memory-based search behav- 
ior (Seamans et al., 1998). Accordingly, we hypothesize that a significant increase in DA release in the $\mathrm{mPFC}$ will occur during accurate recall of the correct location of food sources in a complex spatial environment, independent of the presence or absence of food.

A portion of these data formed part of a poster presentation at the Tenth Annual Conference of the Rotman Research Institute, Toronto, Canada, March 2000.

\section{Materials and Methods}

Apparatus. Foraging tests were conducted on an eight-arm radial maze, consisting of an octagonal center platform $(51 \mathrm{~cm}$ in diameter, arm-toarm) connected to eight equally spaced arms $(40 \times 13 \mathrm{~cm})$. A wooden post $(69 \mathrm{~cm}$ in length) was positioned between two of the arms, and a lever mechanism attached to the top of the post adjusted the tension on the dialysis tubing when a rat moved through the maze. A liquid swivel (Instech 375s) located at the end of the lever was connected directly to the tubing, permitting rats to unimpeded movement.

Surgical procedures and behavioral training. Male Long-Evans rats (280-350 gm) were anesthetized with xylazine and ketamine hydrochloride (7 and $100 \mathrm{mg} / \mathrm{kg}$, i.p., respectively) and stereotaxically implanted with bilateral stainless steel guide cannulas (19 gauge, $15 \mathrm{~mm}$, nitric acid-passivated) $1 \mathrm{~mm}$ below dura, over the $\mathrm{mPFC}(+3.0 \mathrm{~mm}$ anteroposterior and $\pm 0.6 \mathrm{~mm}$ mediolateral from bregma). An additional guide cannula mounted on the skull (training post) with dental acrylic was used to tether the dialysis assembly during training sessions and thereby habituate the animals to this device. A stainless steel coil protected the dialysis tubing from the training post to the liquid swivel.

After a $7 \mathrm{~d}$ postoperative recovery period, rats were food deprived to $85 \%$ of their free-feeding weight. The delayed spatial win-shift (SWSh) task used in the delayed working memory tests is diagrammed in Figure 1. In all experiments described below, rats were trained in the same manner (i.e., 30 min delays, food available during both training and recall phases) regardless of the manipulations that were conducted on microdialysis test days. On the first two days of training, rats were habituated to the maze, and on subsequent daily delayed SWSh training trials, they were habituated to being tethered to the dialysis assembly. On the recall test, rats were permitted as many arm choices as necessary to retrieve the four pellets, and errors were scored as entries into unbaited arms, with criterion performance of one error or less during the test phase. The initial delay between training and recall test phases was $5 \mathrm{~min}$. This delay was extended to $30 \mathrm{~min}$ after a rat achieved criterion performance for 2 consecutive days.

When criterion performance at a 30 min delay was maintained for 3 consecutive days ( $\sim 25-30 \mathrm{~d}$ of training), a microdialysis probe was inserted into the $\mathrm{mPFC}$ via the guide cannula, and a microdialysis experiment was conducted on the following day. In one group of rats $(n=6)$, microdialysis samples were collected during a standard delayed SWSh trial, with food available during both the training phase and recall test phase. As a control for the effect of food consumption on DA efflux, dialysis samples were taken from a second group of rats $(n=6)$ tested with food available during the training phase, but not during the recall phase of the delayed SWSh task. In a third group $(n=7)$, dialysis samples were collected during the standard training phase of the SWSh, and again during recall following an extended $1 \mathrm{hr}$ delay between training and test procedure. In the final experimental group $(n=7)$, dialysis samples were collected during the training phase and again during a recall test after an extended $6 \mathrm{hr}$ delay. During all delay periods in the microdialysis experiments, rats were confined within a wooden box with an open top $(30 \times$ $30 \times 45 \mathrm{~cm}$ ) in the center of the maze.

Microdialysis and HPLC. Microdialysis probes were concentric in design with silica inlet-outlet lines. The active surface consisted of a semi- permeable membrane $2.0 \mathrm{~mm}$ in length (340 $\mu \mathrm{m}$ outer diameter; 65,000 Dalton molecular weight cutoff; Filtral 12; Hospal, Neurnberg, Germany). Probes were flushed continuously at $1 \mu \mathrm{l} / \mathrm{min}$ with a modified Ringer's solution (in mM: $10 \mathrm{NaHPO} 4,1.2 \mathrm{CaCl} 2,3.0 \mathrm{KCl}, 1.0 \mathrm{MgCl}_{2}$, and $147.0 \mathrm{NaCl}, \mathrm{pH} 7.4$ ) using a $2.5 \mathrm{ml}$ gas-tight syringe (Hamilton, Reno, NV) and a syringe pump (model 22; Harvard Apparatus, South Natick, MA). Typical in vivo recoveries of DA conducted at room temperature were $17 \%$ of a standard DA solution.

Analysis of DA content in mPFC dialysates involved separation by reverse-phase high-pressure liquid chromatography (HPLC) and quantification by electrochemical detection (ED). The system consisted of a GBC (Victoria, Australia) LC1120 HPLC pump, a Scientific Systems, Inc. (State College, PA) pulse damper (316 stainless steel model), a Rheodyne manual injector (model 9125; $20 \mu \mathrm{l}$ injection loop), a Princeton (Cranbury, NJ) SPHER $\mathrm{C}_{18}$ column $\left(2 \times 10^{\circ} \mathrm{mm} ; 60 \mathrm{~A} 3 \mu \mathrm{m}\right.$ particles $)$, an Antec Leyden (Leyden, The Netherlands) Links system and an Antec Intro detector with a VT-03 electrochemical flowcell $\left(V_{\text {applied }}=+0.7 \mathrm{~V}\right)$. The mobile phase, a $67 \mathrm{~mm}$ sodium acetate buffer containing $2.15 \mathrm{~mm}$ sodium octyl sulfate and $0.07 \mathrm{~mm}$ EDTA, pH 3.5 (12\% v/v methanol), flowed through the system at $0.2 \mathrm{ml} / \mathrm{min}$. Waters Maxima software was used to collect and analyze chromatographic data. A three-point calibration curve of external DA standards was used to convert the area under the curve of DA peaks into concentration values.

Microdialysis experiments. Microdialysis probes were inserted $(-4.5$ $\mathrm{mm}$ below dura) via guide cannulas into the $\mathrm{mPFC}, 14-16 \mathrm{hr}$ before the start of each dialysis experiment. Implanted animals remained confined overnight in the center of the maze, with a daily ration of food and ad libitum water. Dialysis samples were collected at $5 \mathrm{~min}$ intervals and analyzed immediately with HPLC. Baseline samples were collected while the rat was confined in the center of the maze. After baseline samples had stabilized ( $<5 \%$ change over four consecutive samples), the box was removed, thereby initiating the training phase of the SWSh task in which four open arms on the maze contained food. During the standard $30 \mathrm{~min}$ and extended delay conditions, dialysis samples were obtained at $5 \mathrm{~min}$ intervals throughout the $1 \mathrm{hr}$ delay, the recall test phase, and for $20 \mathrm{~min}$ after completion of the test phase. In the $6 \mathrm{hr}$ delay condition, samples were collected for $40 \mathrm{~min}$ after the training phase, after which no samples were taken until 15 min before the recall test phase. During both the training and test phases for all four conditions, rats were permitted to explore the maze for the entire 5 min sampling period, even if they had retrieved all four food pellets before that period was over. However, only entries into unbaited arms that occurred before retrieving the final pellet were scored as errors.

Data analysis. Neurochemical data were transformed into percentage of change from baseline (i.e., 100\% representing the average concentration of the three samples preceding the final baseline sample). Neurochemical data from each of the four treatment conditions were analyzed 
using a one-way repeated measures ANOVA followed by the Dunnett method of multiple comparisons, using the baseline sample immediately before the training phase as the control sample. Behavioral data from each experiment (errors, latency) for both the training and test phases were analyzed using separate one-way repeated measures ANOVAs with training day (day before test day and test day) as the within-subjects factor. In addition, statistical comparisons across the experimental conditions for both neurochemical and error data were conducted using one-way between-subjects ANOVAs in conjunction with Dunnett's method of multiple comparisons, using the 30 min delay with food condition as the control.

\section{Results}

\section{Basal concentration of DA in microdialysates}

The average concentration of DA in dialysates uncorrected for probe recovery ( $5 \mu$ l samples) collected from the mPFC in the last sample before the training and retrieval phases of the SWSh task were, respectively (in nM): $0.16 \pm 0.02$ and $0.20 \pm 0.02$ for the 30 min delay with food; $0.19 \pm 0.05$ and $0.19 \pm 0.06$ for the $30 \mathrm{~min}$ delay with no food; $0.18 \pm 0.02$ and $0.18 \pm 0.02$ for the $1 \mathrm{hr}$ delay; and $0.24 \pm 0.09$ and $0.25 \pm 0.10$ for the $6 \mathrm{hr}$ delay. A series of one-way ANOVAs conducted on these data revealed that there were no statistically significant differences in basal DA levels before the training versus retrieval phases in all of the experimental conditions (all $F$ values $<2.0$; NS).

\section{Profiles of mesocortical DA release during memory acquisition and retrieval and independence from reward consumption}

Consistent with previous observations of increased DA release during feeding behavior (Ahn and Phillips, 1999), a significant increase in DA efflux $(+78.5 \pm 12 \%)$ in the mPFC was observed during the search for and ingestion of food pellets in the $5 \mathrm{~min}$ acquisition phase $\left(F_{(15,75)}=5.68 ; p<0.001\right.$; Dunnett's, $p<$ $0.01)$. DA levels remained elevated for a further $5 \mathrm{~min}$ period and returned to baseline values for the remaining $25 \mathrm{~min}$ of the delay period. The apparent anticipatory increase in DA efflux in the $\mathrm{mPFC}, 5 \mathrm{~min}$ before the start of the retrieval phase, did not differ significantly from baseline. A second significant increase in DA efflux $(+81.8 \pm 20 \%)$ was observed when rats displayed accurate recall, making only $0.67 \pm 0.3$ errors in their choice of arms that contained food (Fig. 2A).

Importantly, a similar profile of increased DA efflux was observed in a second experiment, although food was not available during the recall phase $\left(F_{(15,75)}=6.42 ; p<0.001\right.$; Dunnett's, $p<$ 0.01 ) (Fig. $2 B$ ). Here it must be noted that although the increase in DA efflux recorded during the recall test of the no food condition was less than that observed during consumption of food pellets during the acquisition phase, a dependent measures $t$ test on these two data points revealed that this difference was not statistically significant $\left(t_{(5)}=1.6 ; p>0.15\right)$. This effect was primarily attributable to one animal who displayed an unusually large $(>200 \%)$ increase in PFC DA efflux during the training phase, whereas the magnitude of DA efflux during the test phase was comparable to the group mean. The absence of food reward during this test day had no effect on accuracy of responding in this separate group of rats that were trained previously with food available during both phases $(0.5 \pm 0.3$ errors $)$. No difference was observed in the number of arm entries required to complete the task on the day before the microdialysis experiment (Fig. $2 A, B$, white bar) compared with the microdialysis test day (black bar) for either the training phase or the recall test phase with either group (both $t$ values $<1.1$; NS). Thus, both the acquisition and
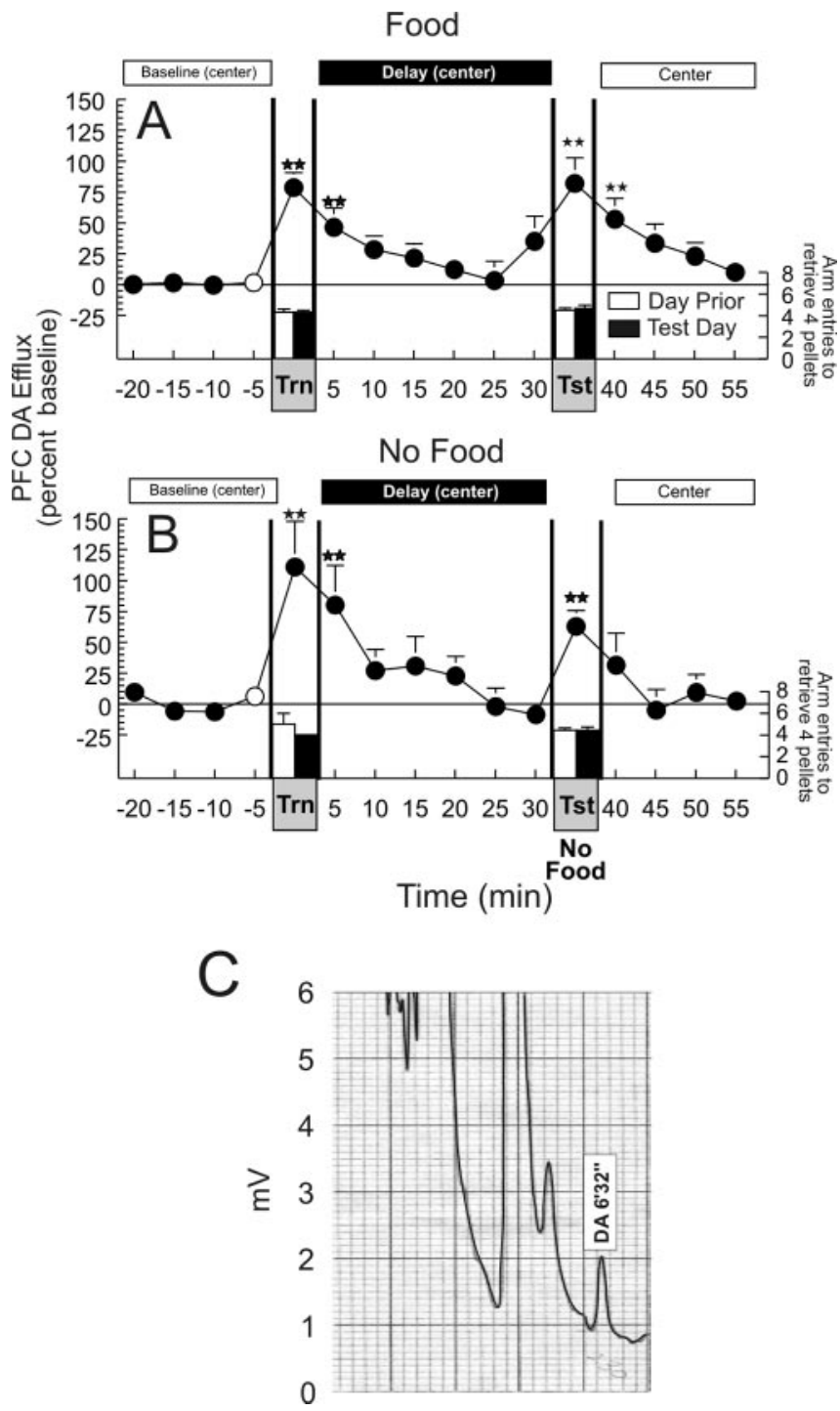

Figure 2. Acquisition and retrieval of information during a delayed SWSh foraging task is associated with an increase in DA efflux in the MPFC. $A$, Changes in DA efflux during (1) baseline (when rats were confined to the center of the maze), (2) the training phase (Trn), (3) a 30 min delay period (when the rats were again confined to the center of the maze), and (4) in the recall test phase (Tst), throughout a delayed SWSh trial with food available during both the training and test phases. $B$, Changes in DA efflux in the mPFC during the same delayed SWSh task without food available during the recall test phase. Circles represent percent change in basal DA extracellular levels in the PFC, and bars represent total number of choices required to retrieve the four food reward pellets during each phase, on the day before (white bars) and the microdialysis test day (black bar). Double stars denote significance at $p<0.01$ versus baseline (white circle). C, Typical chromatogram resulting from HPLC-EC analysis of a $5 \mu$ l sample obtained from the $\mathrm{mPFC}$ by microdialysis over a $5 \mathrm{~min}$ period obtained during the baseline period before the training phase. The DA peak at a retention interval of $6 \mathrm{~min} 32 \mathrm{sec}$ is equivalent to $0.20 \mathrm{~nm}$ of DA.

successful retrieval of trial unique information that is used to guide search behavior is associated with a brief, phasic increase in mesocortical DA release. Moreover, the increase in DA efflux in the PFC observed during retrieval is not caused by the consumption of food. Interestingly, extracellular DA levels return to baseline during the $30 \mathrm{~min}$ delay period. This finding implies that mesocortical DA activity does not play a role in the active maintenance and storage of this information during an extended delay period. In contrast, the storage of information during this delay appears to be mediated by the hippocampus (Floresco et al., 1997, 1999; Floresco and Phillips, 2001). 


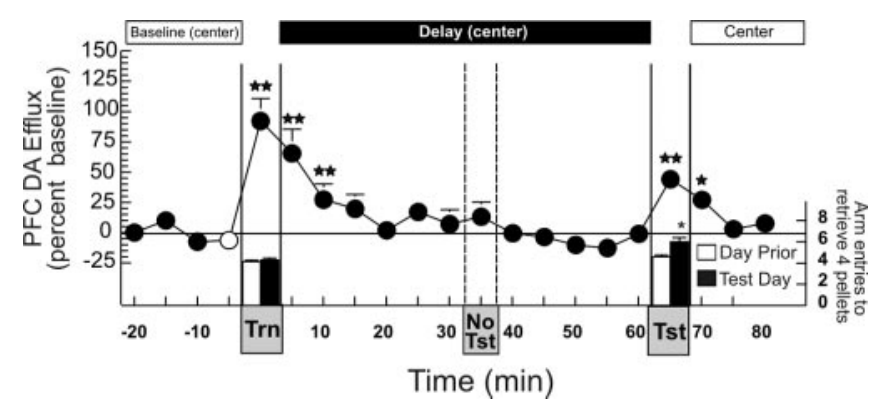

Figure 3. Insertion of an unexpected $1 \mathrm{hr}$ extended delay between training and test phases of the delayed SWSh is associated with a smaller increase in DA efflux in the MPFC and an increase in errors. Changes in DA efflux during (1) baseline (when rats were confined to the center of the maze), (2) the training phase (Trn), (3) a 60 min delay period (when the rats were again confined to the center of the maze), and (4) in the recall test phase (Tst), throughout a delayed SWSh trial with food available during both the training and test phases. Circles represent percent change in basal DA extracellular levels in the PFC, and bars represent total number of choices required to retrieve the four food reward pellets during each phase, on the day before (white bars) and the microdialysis test day (black bar). Dashed lines highlight the period when rats would normally be allowed to explore the maze during the test phase (No Tst). Stars and double stars denote significance at $p<0.05,0.01$ versus baseline (white circle), and asterisk denotes significance at $p<0.05$ versus day before.

\section{Disruptions in memory retrieval and changes in mesocortical} DA release associated with increased task difficulty

Given that accurate recall is facilitated by an increase in DA efflux in the mPFC evoked by stimuli that predict the presence and location of reward, it follows that a disruption of this memory process should be accompanied by a progressive decline in DA efflux. As a test of this hypothesis, we conducted separate experiments in which task difficulty was increased to determine whether a decrement in memory recall was associated with a corresponding decrease in DA levels during the retrieval phase. Accordingly, rats were trained with a standard 30 min delay, but received subsequent tests for recall, in conjunction with brain dialysis, at delays extended unexpectedly to 1 or $6 \mathrm{hr}$. Previous studies have shown that increasing the delay between acquisition and recall degrades memory retrieval during the test phase of the working memory task used here (Packard and White, 1989; Floresco and Phillips, 2001).

Rats in both the 1 and $6 \mathrm{hr}$ delay condition displayed a significant $(p<0.01)$ increase in DA efflux in the mPFC during the training phase. Analysis of these data using a one-way betweensubjects ANOVA confirmed that this increase was comparable to that observed during the training phase in the other treatment conditions $\left(F_{(3,22)}=2.1\right.$; NS). No reliable increase in DA efflux was observed after $30 \mathrm{~min}$, when the rats would normally have been allowed to explore the maze (Fig. 3). Analysis of the neurochemical data using a one-way repeated measures ANOVA revealed that, when rats were released from the chamber in the center of the maze after a $1 \mathrm{hr}$ delay, they still displayed a significant increase in PFC DA release relative to baseline $\left(F_{(20,120)}=\right.$ 8.33; $p<0.001$; Dunnett's, $p<0.01$ ) (Fig. 3). However, the magnitude of DA efflux in the mPFC was blunted significantly $\left(+44 \pm 6 \% ; F_{(3,22)}=6.05 ; p<0.005\right.$; Dunnett's, $\left.p<0.05\right)$, relative to the increase observed in rats that received food after a 30 min delay (Fig. $4 A$ ). Moreover, these rats made significantly more errors during the recall phase than rats tested at a standard 30 min delay $\left(F_{(3,22)}=5.44 ; p<0.01\right.$; Dunnett's, $p<0.05$ ) (Fig. $4 B$ ). This effect was even more pronounced after a $6 \mathrm{hr}$ delay, because there was no significant increase in DA release when these rats searched for food during the recall test phase.
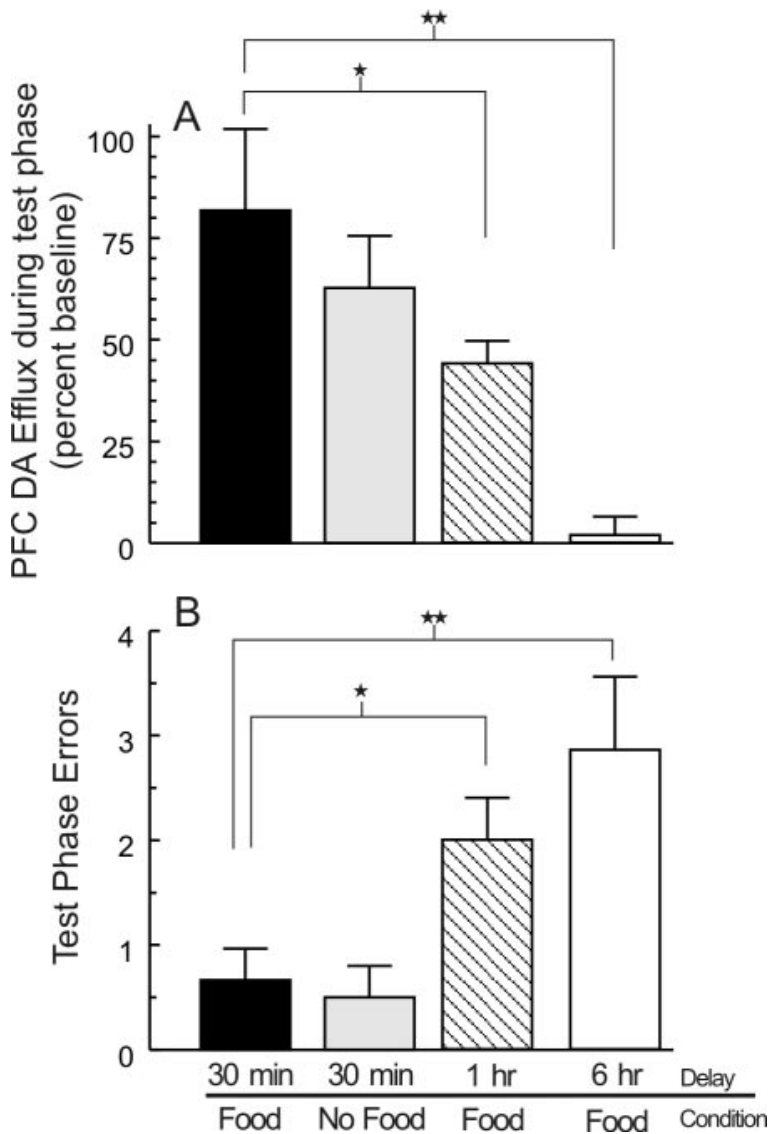

Figure 4. Extended delays of 1 or $6 \mathrm{hr}$, between training and test phases of the delayed SWSh task, are accompanied by reduced levels of DA efflux in the $\mathrm{mPFC}$ during the recall phase and impaired memory for the correct baited arms on the maze. $A$, Change in DA efflux in the MPFC during (1) a recall test in which rats received food at the end of the correct arms ( 30 min delay, black bar), (2), a recall test with no food available (30 min delay, gray bar), (3), a recall test after a $1 \mathrm{hr}$ delay (hatched bar), and (4) a $6 \mathrm{hr}$ extended delay between training and recall test phases (white bar). Rats in the 1 and $6 \mathrm{hr}$ extended delay conditions had significantly lower extracellular levels of DA efflux in the $\mathrm{MPFC}$ during the recall test phase compared with rats in the $30 \mathrm{~min}$ delay condition. $B$, Number of errors committed during the recall test by rats after a standard 30 min delay (black bar), during a recall test with no food available ( 30 min delay, gray bar), and for rats tested for recall after either $1 \mathrm{hr}$ (hatched bar) or $6 \mathrm{hr}$ (white bar) extended delay. Rats in the 1 and $6 \mathrm{hr}$ extended delay condition made significantly more errors than rats in the $30 \mathrm{~min}$ delay condition, and this was associated with lower levels of mesocortical DA efflux. For both $A$ and $B$, stars and double stars denote significance versus $30 \mathrm{~min}$ delay (food) at $p<0.05$, and 0.01, respectively.

Correlational analyses on data collected from all animals in all groups revealed a significant negative correlation between the magnitude of DA release in the PFC during the recall test, and the total number of errors made during this test $\left(r_{(24)}=-0.438 ; p<\right.$ 0.05) (Fig. 5). It is important to note that although the rats searched inefficiently, all food pellets were consumed. The finding that rats retested after a $6 \mathrm{hr}$ delay had no significant increase in DA efflux in the mPFC and made significantly more errors $(p<0.01)$ compared with their own performance at a $30 \mathrm{~min}$ delay on the previous test day, or to a control group (Fig. 4A,B), implies that when task demands were increased (in this case, by extending the delay between acquisition and recall), the tendency to forget relevant spatial location of food was associated with a reduction in the magnitude of DA release in the $\mathrm{mPFC}$. The effect of extended delay on DA efflux and recall of correct arms during memory-based search behavior cannot be attributed to an impairment in motivational or arousal processes or to decreased 


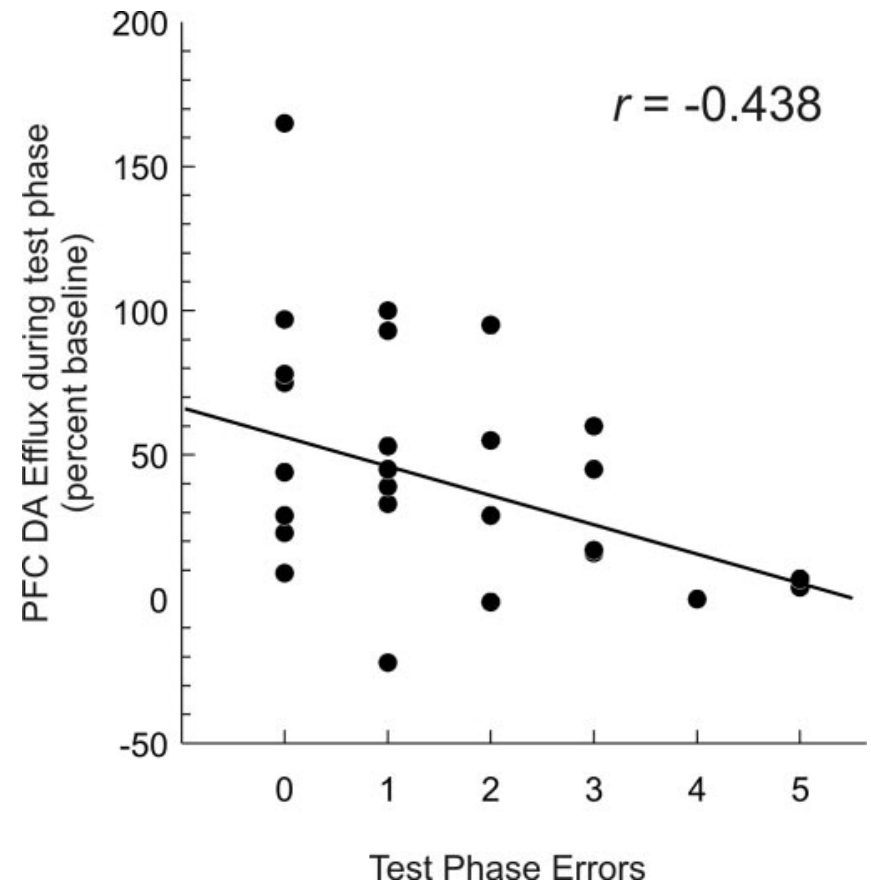

Figure 5. mPFC DA release is negatively correlated with the numbers of errors committed during memory retrieval. Scatterplot displaying the number of errors made during the test phase of the delayed SWSh task ( $x$-axis) as a function of the percentage change in DA efflux during the test phase ( $y$-axis) for rats in all four treatment conditions.

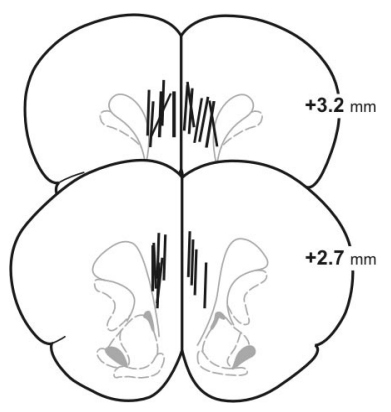

Figure 6. Location of the microdialysis probes in the MPFC. Vertical lines represent length of the $2 \mathrm{~mm}$ dialysis probes. Numbers beside each plate correspond to millimeters from bregma.

ambulation on the maze, because all pellets were consumed and latency to initiate search or average time per choice did not differ statistically between the four treatment groups (both $F$ values $<1.2$; NS).

\section{Histology}

The location of all microdialysis probes is presented in Figure 6. All probes were localized within the prelimbic and infralimbic regions of the $\mathrm{mPFC}$.

\section{Discussion}

The present results show that DA efflux in the mPFC increased in a phasic manner during both the acquisition and retrieval phases of a delayed response task, but returned to baseline levels during the delay period itself. It is unlikely that the increase in mesocortical DA release observed during the training phase plays a role in the encoding of information to be used during the recall test because we have shown previously that inactivation of the $\mathrm{mPFC}$ before the training phase does not disrupt retrieval of information during the recall test 30 min later (Seamans et al., 1995).
Rather, we propose that the subsequent increase in PFC DA efflux that occurs during the recall test is of primary importance in the accurate retrieval of information, a contention supported by the fact that blockade of $\mathrm{D}_{1}$ receptors in the PFC before the test phase disrupts performance (Seamans et al., 1998).

To date, the only evidence linking increased in vivo DA efflux in the PFC to accurate recall during a delayed response task used a 15 min sampling period with a repeated trials design in which monkeys received sequential testing consisting of a $20 \mathrm{~min}$ period of sensory cue guided responding, a 20 min rest period, and a 35 min session of delayed alternation responding, with a $5 \mathrm{sec}$ delay (Watanabe et al., 1997). The present findings extend these earlier results in several important ways. First, the data show that DA efflux is elevated in the mPFC of the rat after an extended delay of $30 \mathrm{~min}$ and in a situation in which spatial memory for the correct location of food must be recalled and incorporated into an accurate plan to search specific arms on the maze. Second, inaccurate recall of the correct locations of food pellets was negatively correlated with the magnitude of DA efflux. Specifically, after a relatively short ( $30 \mathrm{~min}$ ) delay, animals were able to use information stored in memory to locate food efficiently, and their accurate search behavior was associated with a robust increase in mesocortical DA efflux. In contrast, when the delay between acquisition and retrieval of information was extended to a period of up to $6 \mathrm{hr}$, animals tended to forget where food was located on the maze and made more erroneous responses that were coincident with no significant change in the extracellular levels of DA in the mPFC.

The increase in DA efflux in the mPFC was not related to the consumption of a primary reward during the retrieval phase because the magnitude of DA increase was comparable whether or not animals had access to food. Moreover, when the same quantity of food was consumed after an extended delay of $6 \mathrm{hr}$ (when animals had forgotten where food was located), no significant increase in DA efflux was observed in the mPFC. Our finding that the magnitude of DA efflux in the mPFC was not affected by the omission of reward during the recall phase has important implications for the hypothesis that the activity of DA neurons encodes information about conditioned reward-predicting stimuli, and "prediction errors" of primary reward (Schultz, 1998). DA neurons in primates initially display increased firing whenever rewards are presented unexpectedly, as is often the case during the initial phase of learning. Responding subsequently shifts to stimuli that predict reward and the activity of DA neurons is inhibited when reward is omitted (Ljungberg et al., 1992; Schultz et al., 1993; Schultz, 1998). The sustained elevation of DA during a 5 min period of reward omission observed in the present study stands in contrast to this inhibition of DA neuron activity (Hollerman and Schultz, 1998). Thus, whereas omission of an expected reward may be associated with a decrease in firing of individual DA neurons (Schultz, 1998), it is apparent that implementation of an accurate search strategy for food can override this effect and evoke increases in mesocortical DA release. Reconciliation of microdialysis data from the rat mPFC with electrophysiological data from the primate mesencephalon may come from the recent observation of a sustained increase in DA neuron activity related to uncertainty about motivationally relevant stimuli (Fiorillo et al., 2003). The uncertainty accompanying the search for food in a complex environment may serve as an optimal condition for evoking tonic firing of DA neurons, which in turn would increase the efflux of DA in terminal regions such as the mPFC.

Previous microdialysis studies of feeding behavior have 
shown that consumption of food or exposure to food odors during an anticipatory phase of feeding behavior is associated with a robust increase in PFC DA efflux (Bassareo and Di Chiara, 1997; Ahn and Phillips 1999, 2002). Given these observations, how can they be reconciled with the present finding that rats exposed to the $6 \mathrm{hr}$ delay condition searched actively for food on the maze, consumed pellets once they were found, but showed no discernible increase in DA efflux in the PFC? Our recent studies of changes in DA efflux in the mPFC of the rat during sensoryspecific satiety indicate that although sensory stimuli associated with a specific type of food can elicit a significant increase in DA efflux before and during its consumption, this relationship can be modified by recent experience such as feeding to satiety that can devalue this food (Ahn and Phillips, 2002). In a similar manner, contextual stimuli associated with the location of food may also have the capacity to activate the release of DA in the mPFC. Under circumstances in which specific associations between spatial cues and food were degraded by extended delays, as in the $6 \mathrm{hr}$ condition in the present study, re-exposure to the maze may fail to elicit increased efflux of DA in the mPFC. This explanation emphasizes the probable relationship between memory-guided search behavior for food and mesocortical dopaminergic activity, rather than consumption of a primary reward.

As noted above, when an extended delay was inserted between the acquisition and retrieval phases of this delayed response task, rats made significantly more errors compared with their performance at a 30 min delay, and this increase in errors was negatively correlated with a decrease in the relative change of DA release in the $\mathrm{mPFC}$. The question remains regarding whether the increase in retrieval errors during the extended delay conditions can be attributed to reduced levels of mesocortical DA or whether the reduction in DA efflux in the PFC is caused by the animals forgetting. Resolution of this question comes from neuropharmacological data from our laboratory (Floresco and Phillips, 2001). Using a similar, extended delay protocol, we found the disruption in working memory caused by an extended delay can be alleviated in part by infusion of a $D_{1}$ receptor agonist (SKF81297) into the mPFC before retrieval. The fact that pharmacological stimulation of $\mathrm{D}_{1}$ receptors in the $\mathrm{mPFC}$ can restore working memory that is disrupted at a time when mesocortical DA release would be perturbed (i.e., by an extended delay) further supports the contention that the magnitude of DA release and the accuracy of working memory are causally linked. This finding, in combination with the pattern of results observed across the three delay conditions in the present study, indicate that the magnitude of DA efflux in the mPFC during the retrieval phase of a delayed response task is predictive of the accuracy of recall of baited arms, with lower levels of DA efflux associated with poorer performance.

Rather than construing mesocortical DA efflux as an index of the presence or absence of primary reward, we propose that it is a neurochemical correlate of cortical arousal that can facilitate the recall of information regarding the availability and location of a reward. In the context of working memory, a phasic increase in DA efflux in the mPFC during recall would ensure an appropriate level of $\mathrm{DA} \mathrm{D}_{1}$ receptor activation essential for the retrieval of trial-unique information by executive processes in the $\mathrm{mPFC}$, which in turn guides behavioral selection during delayed response tasks (Sawaguchi et al., 1990; Sawaguchi and GoldmanRakic, 1994; Williams and Goldman-Rakic, 1995; Seamans et al., 1998; Robbins, 2000). Although the time scales differ, the relationship between the magnitude of DA efflux and the accuracy of correct choices during recall tests parallels findings in electro- physiological studies of single-unit activity in the PFC during delayed memory tasks. In these experiments, robust increases in neural activity during either the delay period or responding are associated with accurate memory, whereas low levels of firing are accompanied by erroneous responses (Goldman-Rakic, 1992, 1995; Pratt and Mizumori, 2001).

Of relevance to the present study are anatomical data indicating that pyramidal neurons in the $\mathrm{mPFC}$ (including those which receive hippocampal inputs) send glutamatergic projections to DA neurons in the ventral tegmental area, which in turn are connected in a reciprocal manner to the PFC (Christie et al., 1985; Jay et al., 1995; Carr and Sesack, 2000; Floresco and Grace, 2003). These findings suggest that both the increase in task-related firing of PFC neurons and the enhanced efflux of mesocortical DA may be interrelated. Specifically, increased neural activity in the mPFC essential for efficient search behavior may in turn initiate corresponding changes in PFC DA efflux. Support for this conjecture is provided by data from our laboratory (Taepavarapruk and Phillips, 2001) and others (Gurden et al., 2000) confirming that stimulation of hippocampal afferents can increase DA efflux in the $\mathrm{mPFC}$, an effect that is mediated by reciprocal connections between the PFC and ventral tegmental area (Taepavarapruk and Phillips, 2001). In light of these data, we propose that activity within a neural circuit linking the ventral hippocampus to the $\mathrm{mPFC}$, which in turn engages the mesocorticolimbic DA projection, forms an essential substrate for working memory retrieval across short and long delays. The present data also imply that accuracy of memory-based search behavior is dependent on an appropriate level of DA in the $\mathrm{MPFC}$, and that failure to recall the correct locations of food (as may occur when task demands increase) may be attributed in part to a perturbation in DA release that normally occurs during retrieval. Accordingly, drugs that serve as DA agonists, especially DA $\mathrm{D}_{1}$ agonists (Floresco and Phillips, 2001), may provide pharmacotherapy for certain forms of dementia related to loss of function in the PFC.

\section{References}

Ahn S, Phillips AG (1999) Dopaminergic correlates of sensory-specific satiety in the medial prefrontal cortex and the nucleus accumbens of the rat. J Neurosci 19:RC29(1-6).

Ahn S, Phillips AG (2002) Modulation by central and basolateral amygdalar nuclei of dopaminergic correlates of feeding to satiety in the rat nucleus accumbens and medial prefrontal cortex. J Neurosci 22:10958-10965.

Arnsten AFT (1998) Catecholamine modulation of prefrontal cortical cognitive function. Trends Cogn Sci 2:436-447.

Baddeley AD (1986) Working memory. Oxford: Clarendon.

Baddeley AD (1992) Working memory. Science 255:556-5590.

Bassareo V, Di Chiara G (1997) Differential influence of associative and nonassociative learning mechanisms on the responsiveness of prefrontal and accumbal dopamine transmission to food stimuli in rats fed ad libitum. J Neurosci 17:851-861.

Carr DB, Sesack SR (2000) Projections from the rat prefrontal cortex to the ventral tegmental area: target specificity in the synaptic associations with mesoaccumbens and mesocortical neurons. J Neurosci 20:3864-3873.

Christie MJ, Bridge S, James LB, Beart PM (1985) Excitotoxin lesions suggest an aspartatergic projection from rat medial prefrontal cortex to ventral tegmental area. Brain Res 333:169-172.

Fiorillo CD, Tobler PN, Schultz W (2003) Discrete coding of reward probability and uncertainty by dopamine neurons. Science 299:1898-1902.

Floresco SB, Phillips AG (2001) Delay-dependent modulation of memory retrieval by infusion of a dopamine D1 agonist into the rat medial prefrontal cortex. Behav Neurosci 115:934-939.

Floresco SB, Grace AA (2003) Gating of hippocampal-evoked activity in prefrontal cortical neurons by inputs from the mediodorsal thalamus and ventral tegmental area. J Neurosci 23:3930-3943.

Floresco SB, Seamans JK, Phillips AG (1997) Selective roles for hippocam- 
pal, prefrontal cortical, and ventral striatal circuits in radial-arm maze tasks with or without a delay. J Neurosci 17:1880-1890.

Floresco SB, Braaksma DN, Phillips AG (1999) Thalamic-cortical-striatal circuitry subserves working memory during delayed responding on a radial arm maze. J Neurosci 19:11061-11071.

Goldman-Rakic PS (1992) Working memory and the mind. Sci Am 267:110-117.

Goldman-Rakic PS (1995) Cellular basis of working memory. Neuron 14:477-485.

Gurden H, Takita M, Jay TM (2000) Essential role of D1 but not D2 Receptors in the NMDA receptor-dependent long-term potentiation at hippocampal-prefrontal cortex synapses in vivo. J Neurosci 20:RC106(1-5).

Hollerman JR, Schultz W (1998) Dopamine neurons report an error in the temporal prediction of reward during learning. Nat Neurosci 1:304-309.

Jay TM, Glowinski J, Thierry AM (1995) Inhibition of hippocampoprefrontal cortex excitatory responses by the mesocortical DA system. NeuroReport 6:1845-1848.

Ljungberg T, Apicella P, Schultz W (1992) Responses of monkey dopamine neurons during learning of behavioral reactions. J Neurophysiol 67:145-163.

Mizumori SJ, Channon V, Rosenzweig MR, Bennett EL (1987) Short- and long-term components of working memory in the rat. Behav Neurosci 101:782-789.

Packard MG, White NM (1989) Memory facilitation produced by dopamine agonists: role of receptor subtype and mnemonic requirements. Pharmacol Biochem Behav 33:511-518.

Pratt WE, Mizumori SJ (2001) Neurons in rat medial prefrontal cortex show anticipatory rate changes to predictable differential rewards in a spatial memory task. Behav Brain Res 123:165-183.

Robbins TW (2000) Chemical neuromodulation of frontal-executive functions in humans and other animals. Exp Brain Res 133:130-138.

Sawaguchi T, Matsumura M, Kubota K (1990) Effects of dopamine antag- onists on neuronal activity related to a delayed response task in monkey prefrontal cortex. J Neurophysiol 63:1401-1412.

Sawaguchi T, Goldman-Rakic PS (1994) The role of D1-dopamine receptor in working memory: local injections of dopamine antagonists into the prefrontal cortex of rhesus monkeys performing an oculomotor delayedresponse task. J Neurophysiol 71:515-528.

Schultz W (1998) Predictive reward signal of dopamine neurons. J Neurophysiol 80:1-27.

Schultz W, Apicella P, Ljungberg (1993) Responses of monkey dopamine neurons to reward and conditioned stimuli during successive steps of learning a delayed response task. J Neurosci 13:900-913.

Seamans JK, Floresco SB, Phillips AG (1995) Functional differences between the prelimbic and anterior cingulate regions of rat prefrontal cortex. Behav Neurosci 109:1063-1073.

Seamans JK, Floresco SB, Phillips AG (1998) $D_{1}$ receptor modulation of hippocampal-prefrontal cortical circuits integrating spatial memory with executive functions in the rat. J Neurosci 18:1613-1621.

Taepavarapruk P, Phillips AG (2001) Role of the prefrontal cortex and ventral tegmental area in mediating release of dopamine in the nucleus accumbens evoked by stimulation of the ventral subiculum In: Monitoring molecules in neuroscience. Proceedings of the $9^{\text {th }}$ international conference on in vivo methods (O'Connor WT, Lowry JP, O'Connor JJ, and O’Neill RD, eds), pp 223-224. Dublin, Ireland: University College of Dublin.

Watanabe M, Kodama T, Hikosaka K (1997) Increase of extracellular dopamine in primate prefrontal cortex during a working memory task. J Neurophysiol 78:2795-2798.

Williams GV, Goldman-Rakic PS (1995) Modulation of memory fields by dopamine D1 receptors in prefrontal cortex. Nature 376:572-575.

Zahrt J, Taylor JR, Mathew RG, Arnsten AF (1997) Supranormal stimulation of D1 dopamine receptors in the rodent prefrontal cortex impairs spatial working memory performance. J Neurosci 17:8528-8535. 